\title{
The Structure and Number of Global Roundings of a Graph
}

\author{
Tetsuo Asano \\ School of Information Science, Japan Advanced Institute of Science and \\ Technology, Tatsunokuchi, Japan \\ Naoki Katoh \\ Graduate School of Engineering, Kyoto University, Kyoto, Japan. \\ Hisao Tamaki \\ School of Science and Technology, Meiji University, Kawasaki, Japan \\ Takeshi Tokuyama* \\ Graduate School of Information Sciences, Tohoku University, Sendai, Japan.
}

\begin{abstract}
Given a connected weighted graph $G=(V, E)$, we consider a hypergraph $H_{G}=$ $\left(V, \mathcal{P}_{G}\right)$ corresponding to the set of all shortest paths in $G$. For a given real assignment a on $V$ satisfying $0 \leq \mathbf{a}(v) \leq 1$, a global rounding $\alpha$ with respect to $H_{G}$ is a binary assignment satisfying that $\left|\sum_{v \in F} \mathbf{a}(v)-\alpha(v)\right|<1$ for every $F \in \mathcal{P}_{G}$. We conjecture that there are at most $|V|+1$ global roundings for $H_{G}$, and also the set of global roundings is an affine independent set. We give several positive evidences for the conjecture.
\end{abstract}

Key words: Combinatorics, Rounding, Discrepancy, Graph, Hypergraph 1991 MSC: 05C65, 05C90, 68Q20, 68Q25

\footnotetext{
* Corresponding author Email addresses: t-asano@jaist.ac.jp ( Tetsuo Asano), naoki@archi.kyoto-u.ac.jp (Naoki Katoh), tamaki@cs.meiji.ac.jp (Hisao Tamaki), tokuyama@dais.is.tohoku.ac.jp (Takeshi Tokuyama).
} 


\section{Introduction}

Given a real number $a$, an integer $k$ is a rounding of $a$ if the difference between $a$ and $k$ is strictly less than 1 , or equivalently, if $k$ is the floor $\lfloor a\rfloor$ or the ceiling $\lceil a\rceil$ of $a$. We extend this usual notion of rounding into that of global rounding on hypergraphs as follows.

Let $H=(V, \mathcal{F})$, where $\mathcal{F} \subset 2^{V}$, be a hypergraph on a set $V$ of $n$ nodes. Given a real valued function a on $V$, we say that an integer valued function $\alpha$ on $V$ is a global rounding of a with respect to $H$, if $w_{F}(\alpha)$ is a rounding of $w_{F}(\mathbf{a})$ for each $F \in \mathcal{F}$, where $w_{F}(f)$ denotes $\sum_{v \in F} f(v)$. We assume in this paper that the hypergraph contains all the singleton sets as hyperedges; thus, $\alpha(v)$ is a rounding of $\mathbf{a}(v)$ for each $v$, and we can restrict our attention to the case where the ranges of $\mathbf{a}$ and $\alpha$ are $[0,1]$ and $\{0,1\}$ respectively.

This notion of global roundings on hypergraphs is closely related to that of discrepancy of hypergraphs $[6,10,11,4]$. Given $\mathbf{a}$ and $\mathbf{b} \in[0,1]^{V}$, define the discrepancy $D_{H}(\mathbf{a}, \mathbf{b})$ between them on $H$ by

$$
D_{H}(\mathbf{a}, \mathbf{b})=\max _{F \in \mathcal{F}}\left|w_{F}(\mathbf{a})-w_{F}(\mathbf{b})\right|
$$

The supremum $\sup _{\mathbf{a} \in[0,1]^{V}} \min _{\alpha \in\{0,1\}^{V}} D_{H}(\mathbf{a}, \alpha)$ is called the linear (or inhomogeneous) discrepancy of $H$, and it is a quality measure of approximability of a real vector with an integral vector to satisfy constraints given by the linear system corresponding to $H$.

Thus, the set of global roundings of $\mathbf{a}$ is the set of integral points in the open unit ball around a where the distance is measured by the discrepancy $D_{H}$. It is known that the open ball always contains an integral point for any "input" $\mathbf{a}$ if and only if the hypergraph is unimodular (see $[4,7]$ ). This fact is utilized in digital halftoning applications [1,2]. It is NP-hard to decide whether the ball is empty (i.e. containing no integral point) or not even for some very simple hypergraphs [3].

In this paper, we are interested in the maximum number $\nu(H)$ of integral points in an open unit ball under the discrepancy distance.

This direction of research was initiated by Sadakane et al.[13] where the authors discovered a surprising fact that $\nu\left(I_{n}\right) \leq n+1$ where $I_{n}$ is a hypergraph on $V=\{1,2, \ldots, n\}$ with edge set $\{[i, j] ; 1 \leq i \leq j \leq n\}$ consisting of all subintervals of $V$. We can also see that $\nu(H) \geq n+1$ for any hypergraph $H$ : if we let $\mathbf{a}(v)=\epsilon$ for every $v$, where $\epsilon<1 / n$, then any binary assignment on $V$ that assigns 1 to at most one vertex is a global rounding of $H$, and hence $\nu(H) \geq n+1$. 
Given this discovery, it is natural to ask for which class of hypergraphs this property $\nu(H)=n+1$ holds. The understanding of such classes may well be related to algorithmic questions mentioned above. In fact, Sadakane et al. give an efficient algorithm to enumerate all the global roundings of a given input on $I_{n}$.

In this paper, we show that $\nu(H)=n+1$ holds for a considerably wider class of hypergraphs. Given a connected $G$ in which edges are possibly weighted by a positive value, we define a shortest-path hypergraph $H_{G}$ generated by $G$ as follows: a set $F$ of vertices of $G$ is an edge of $H_{G}$ if and only if $F$ is the set of vertices of some shortest path ${ }^{1}$ in $G$ with respect to the given edge weights. In this notation, $I_{n}=H_{P_{n}}$ for the path $P_{n}$ on $n$ vertices. Note that we permit more than one shortest path between a pair of nodes if they have the same weight. We give several basic properties of the structure of a set of global roundings for $H_{G}$, and prove the following theorem:

Theorem $1.1 \nu\left(H_{G}\right)=n+1$ holds for the shortest-path hypergraph $H_{G}$, if $G$ is a tree, a cycle, a tree of cycles, an unweighted mesh, or an unweighted $k$-tree.

Based on the positive evidence above and some failed attempts in creating counterexamples, we conjecture that the result holds for general connected graphs.

Conjecture $1.2 \nu\left(H_{G}\right)=n+1$ for any connected graph $G$ with $n$ nodes.

\section{Preliminaries}

We start with the following easy observations:

Lemma 2.1 For hypergraphs $H=(V, \mathcal{F})$ and $H^{\prime}=\left(V, \mathcal{F}^{\prime}\right)$ such that $\mathcal{F} \subset \mathcal{F}^{\prime}$, $\nu(H) \geq \nu\left(H^{\prime}\right)$.

Definition $1 A$ set $A$ of binary functions on $V$ is called $H$-compatible if, for each pair $\alpha$ and $\beta$ in $A,\left|w_{F}(\alpha)-w_{F}(\beta)\right| \leq 1$ holds for every hyperedge $F$ of $H$.

Lemma 2.2 For a given input real vector $\mathbf{a}$, the set of global roundings with respect to $H$ is $H$-compatible.

Proof: Suppose that $\alpha$ and $\beta$ are two different global roundings of an input a with respect to a hypergraph $H$. We have $\left|w_{F}(\alpha)-w_{F}(\beta)\right| \leq \mid w_{F}(\mathbf{a})-$

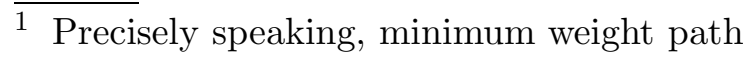


$w_{F}(\alpha)|+| w_{F}(\mathbf{a})-w_{F}(\beta) \mid<2$. Since the value must be integral, we have the lemma.

Thus, any set of global roundings of a given input is an $H$-compatible set. Conversely, we can prove that any $H$-compatible set is a set of global roundings for a suitable input vector.

Lemma 2.3 Given an $H$-compatible set $A=\left\{\alpha_{1}, \alpha_{2}, \ldots, \alpha_{m}\right\}, A$ is a set of global roundings of the center of gravity $\mathbf{g}=\frac{1}{m} \sum_{i=1}^{m} \alpha_{i}$ of $A$.

Proof: It is clear that $\mathbf{g} \in[0,1]^{V}$. For each $\alpha_{i}$, the set $P_{i}$ of vectors $\mathbf{x}$ in $[0,1]^{V}$ satisfying that $D_{H}\left(\mathbf{x}, \alpha_{i}\right)<1$ is a convex set (indeed, it is a convex polytope). Also, $\alpha_{j}$ is in the closure of $P_{i}$ for all $j \neq i$. Thus, $\mathbf{g}_{i}=\frac{1}{m-1} \sum_{j \neq i} \alpha_{j}$ is in the closure of $P_{i}$, and $\mathbf{g}=\frac{1}{m}\left((m-1) \mathbf{g}_{i}+\alpha_{i}\right)$ is in the interior of $P_{i}$. Thus, $D_{H}\left(\mathbf{g}, \alpha_{i}\right)<1$, and $\alpha_{i}$ is a global rounding of $\mathbf{g}$.

Therefore, $\nu(H)$ equals the largest cardinality of an $H$-compatible set. The definition of an $H$-compatible set does not include the input vector $\mathbf{a}$, and facilitates the combinatorial analysis.

For a vector $\mathbf{q}$ in the $n$-dimensional real space $\mathcal{R}^{n}, \tilde{\mathbf{q}}$ is the vector in $\mathcal{R}^{n+1}$ obtained by appending 1 as the last coordinate value: i.e., $\tilde{\mathbf{q}}=\left(q_{1}, q_{2}, \ldots, q_{n}, 1\right)$ if $\mathbf{q}=\left(q_{1}, q_{2}, \ldots, q_{n}\right)$. Vectors $\mathbf{q}_{1}, \mathbf{q}_{2}, \ldots \mathbf{q}_{s}$ are called affine independent in $\mathcal{R}^{n}$ if $\tilde{\mathbf{q}_{1}}, \tilde{\mathbf{q}_{2}}, \ldots, \tilde{\mathbf{q}_{s}}$ are linearly independent in $\mathcal{R}^{n+1}$. If every $H$-compatible set is an affine independent set regarded as a set of vectors in the $n$-dimensional space, we call $H$ satisfies the affine independence property.

Conjecture 2.4 For any connected graph $G, H_{G}$ satisfies the affine independence property.

It is clear that Conjecture 2.4 implies Conjecture 1.2 .

\section{Properties of Compatible Sets of General Graphs}

For a binary assignment $\alpha$ on $V$ and a subset $X$ of $V,\left.\alpha\right|_{X}$ denotes the restriction of $\alpha$ on $X$. Let $V=X \cup Y$ be a partition of $V$ into nonintersecting subsets $X$ and $Y$ of vertices. For binary assignments $\alpha$ on $X$ and $\beta$ on $Y$, $\alpha \oplus \beta$ is a binary assignment on $V$ obtained by concatenating $\alpha$ and $\beta$ : That is, $\alpha \oplus \beta(v)=\alpha(v)$ if $v \in X$, otherwise it is $\beta(v)$.

The following is a key lemma for our theory.

Lemma 3.1 Let $G=(V, E)$ be a connected graph, and let $V=X \cup Y$ be a 
partition of $V$. Let $\alpha_{1}$ and $\alpha_{2}$ be different assignments on $X$ and let $\beta_{1}$ and $\beta_{2}$ be different assignments on $Y$. Then, the set $\mathcal{F}=\left\{\alpha_{1} \oplus \beta_{1}, \alpha_{1} \oplus \beta_{2}, \alpha_{2} \oplus \beta_{1}\right.$, $\left.\alpha_{2} \oplus \beta_{2}\right\}$ cannot be $H_{G^{-}}$compatible.

Proof: Consider $x \in X$ satisfying $\alpha_{1}(x) \neq \alpha_{2}(x)$ and $y \in Y$ satisfying $\beta_{1}(y) \neq \beta_{2}(y)$. We choose such $x$ and $y$ with the minimum shortest path length. Thus, on each internal node of a shortest path $\mathbf{p}$ from $x$ to $y$, all four assignments in $\mathcal{F}$ take the same value. Without loss of generality, we assume $\alpha_{1}(x)=\beta_{1}(y)=0$ and $\alpha_{2}(x)=\beta_{2}(y)=1$. Then, $w_{\mathbf{p}}\left(\alpha_{2} \oplus \beta_{2}\right)=w_{\mathbf{p}}\left(\alpha_{1} \oplus \beta_{1}\right)+2$, and hence violate the compatibility.

Corollary 3.2 Let $A$ be an $H_{G}$-compatible set, and let $\left.A\right|_{X}$ and $\left.A\right|_{Y}$ be the set obtained by restricting assignments of $A$ to $X$ and $Y$, respectively, for a partition $(X, Y)$ of $V$. If $\left.A\right|_{X}$ and $\left.A\right|_{Y}$ have $\nu_{X}$ and $\nu_{Y}$ elements, respectively, and $\nu_{X} \geq \nu_{Y},|A| \leq \min \left\{\nu_{Y}\left(\nu_{Y}-1\right) / 2+\nu_{X}, \nu_{Y} \sqrt{\nu_{X}}+\nu_{X}\right\}$.

Proof: If we construct a bipartite graph with node sets corresponding to $\left.A\right|_{X}$ and $\left.A\right|_{Y}$, in which two nodes $\left.\alpha \in A\right|_{X}$ and $\left.\beta \in A\right|_{Y}$ are connected by an edge if $\alpha \oplus \beta \in A$, Lemma3.1 implies the $K_{22}$-free property of the graph. Thus, the corollary follows from a famous result in extremal graph theory ([5], Lemma $9)$.

If we consider the case where $|X|=n-1$ and $|Y|=1$, we have $|A| \leq 1+\nu_{X}$, since $\nu_{Y} \leq 2$. However, although the recursive formula $f(n) \leq 1+f(n-1)$ gives a linear upper bound of $f(n)$, this does not imply that $\nu(G)=O(n)$, since the restriction $\left.A\right|_{X}$ is not always an $H_{G^{\prime}}$-compatible set where $G^{\prime}$ is the induced subgraph by $X$ of $G$.

The affine independence of an $H$-compatible set $A=\left\{\alpha_{1}, \alpha_{2}, \ldots, \alpha_{m}\right\}$ means that any linear relation $\sum_{1 \leq i \leq m} c_{i} \alpha_{i}=0$ satisfying that $\sum_{1 \leq i \leq m} c_{i}=0$ implies that $c_{i}=0$ for $1 \leq i \leq m$. We can prove its special case as follows: ${ }^{2}$

Proposition 3.3 If $\alpha, \beta, \alpha^{\prime}$, and $\beta^{\prime}$ are mutually distinct elements of an $H_{G^{-}}$ compatible set for some graph $G$, then it cannot happen that $\alpha-\beta=\alpha^{\prime}-\beta^{\prime}$.

Proof: Assume on the contrary that $\alpha-\beta=\alpha^{\prime}-\beta^{\prime}$. Let $X$ be the subset of $V$ consisting of $u$ satisfying $\alpha(u)=\beta(u)$, and let $Y$ be its complement in $V$. Let $\alpha=\xi \oplus \eta$, where $\xi$ and $\eta$ are the parts of $\alpha$ on $X$ and $Y$, respectively. Thus, $\beta=\xi \oplus \bar{\eta}$, where $\bar{\eta}$ is obtained by flipping all the entries of $\eta$.

Let $\alpha^{\prime}=\xi^{\prime} \oplus \eta^{\prime}$. Then, since $\alpha-\beta=\alpha^{\prime}-\beta^{\prime}, \beta^{\prime}=\xi^{\prime} \oplus \bar{\eta}^{\prime}$. Moreover, $\eta-\bar{\eta}$ and $\eta^{\prime}-\bar{\eta}^{\prime}$ are vectors whose entries are 1 and -1 because of the definition of $Y$, and hence $\eta-\bar{\eta}=\eta^{\prime}-\bar{\eta}^{\prime}$ implies that $\eta=\eta^{\prime}$.

$\overline{2}$ This fact was suggested by Günter Rote. 
Thus, all of $\xi \oplus \eta, \xi \oplus \bar{\eta}, \xi^{\prime} \oplus \eta$, and $\xi^{\prime} \oplus \bar{\eta}$ are in $A$; this contradicts with Lemma 3.1.

\section{Graphs for Which The Conjectures Hold}

\subsection{Graphs with Path-Preserving Ordering}

Given a connected graph $G=(V, E)$, consider an ordering $v_{1}, v_{2}, \ldots, v_{n}$ of nodes of $V$. Let $V_{i}=\left\{v_{1}, v_{2}, \ldots, v_{i}\right\}$, and let $G_{i}$ be the induced subgraph of $G$ by $V_{i}$. The ordering is path-preserving if $G_{i}$ is connected for each $i$, and every shortest path in $G_{i}$ is a shortest path in $G$. It is clear that a tree with arbitrary edge lengths and a complete graph with a uniform edge length have path-preserving orderings. More generally, a $k$-tree with a uniform edge length has a path-preserving ordering by its definition. A $d$-dimensional mesh, where each edge has unit length, is also a typical example.

Theorem 4.1 If $G$ has a path-preserving ordering, $H_{G}$ satisfies the affine independence property.

Proof: We prove the statement by induction on $n=|V|$. If $n=1$, the statement is trivial, since $(0,1)$ and $(1,1)$ are linearly independent. If $G$ has a path-preserving ordering, it gives a path-preserving ordering for $G_{n-1}$ that has $n-1$ nodes. Thus, from the induction hypothesis, we assume that any $H_{G_{n-1}}$ compatible set of binary assignments is an affine independent set. Let $\pi$ be the restriction map from $\{0,1\}^{V}$ to $\{0,1\}^{V_{n-1}}$ defined by $\pi(\alpha)(v)=\alpha(v)$ for every $v \in V_{n-1}$. Let $A$ be an $H_{G^{-}}$compatible set, and let $\pi(A)=\{\pi(\alpha): \alpha \in A\}$ be the set obtained by restricting $A$ to $V_{n-1}$ and removing the multiplicities. The set $\pi(A)$ must be an $H_{G_{n-1}}$-compatible set: otherwise, there must be a shortest path in $G_{n-1}$ violating the compatibility condition for $A$, which cannot happen since the path is also a shortest path in $G$.

For each $\beta \in \pi(A)$, let $\beta \oplus 0$ and $\beta \oplus 1$ be its extension in $\{0,1\}^{V}$ obtained by assigning 0 and 1 to $v_{n}$, respectively. Naturally, $\pi^{-1}(\beta)$ is a subset of $\{\beta \oplus$ $0, \beta \oplus 1\}$. For any two different assignments $\beta$ and $\gamma$ in $\pi(A)$, it cannot happen that all of $\beta \oplus 0, \beta \oplus 1, \gamma \oplus 0$, and $\gamma \oplus 1$ are in $A$. Indeed, this is a special case of Lemma 3.1 for $X=V_{n-1}$ and $Y=\left\{v_{n}\right\}$. Thus, there is at most one rounding in $\pi(A)$ satisfying that its inverse image by $\pi$ contains two elements.

List the elements of $A$ as $\alpha_{1}, \ldots, \alpha_{k}$ where $\alpha_{1}=\beta \oplus 0$ and $\alpha_{2}=\beta \oplus 1$ for some $\beta \in \pi(A)$. Suppose a linear relation $\sum_{1 \leq i \leq k} c_{i} \alpha_{i}=0$ holds with $\sum_{1 \leq i \leq k} c_{i}=0$. By the induction hypothesis that $\pi(A)$ is affine independent, we have $c_{1}+c_{2}=0$ and $c_{i}=0$ for $3 \leq i \leq k$. Because of the last components 
of the vectors, it follows that $c_{1}=c_{2}=0$ as well.

Corollary 4.2 For a connected graph $G$, if we consider the hypergraph $H$ associated with the set of all paths in $G$ (irrespective of their lengths), $H$ satisfies the affine independence property.

Proof: Consider a spanning tree $T$ of $G$. Then the hypergraph associated with the set of all paths in $G$ has the same node set as $H$, and its hyperedge set is a subset of that of $H$. Hence, it suffices to prove the statement for $T$, which has a path-preserving ordering. Every path in $T$ is a shortest path in $T$; hence, the set is $H_{T}$-compatible, and consequently, affine independent.

\subsection{Connecting Two Graphs}

An edge $e$ in a connected graph $G$ is called a bridge if the graph is separated into two connected components by removing $e$ from $G$.

Theorem 4.3 Suppose that a graph $G$ has a bridge e separating $G-\{e\}$ into two connected components $G_{1}$ and $G_{2}$. Then, $\nu(G) \leq \nu\left(G_{1}\right)+\nu\left(G_{2}\right)-1$. Moreover, if both of $H_{G_{1}}$ and $H_{G_{2}}$ satisfy the affine independence property, $H_{G}$ does.

Proof: Consider an $H_{G^{-}}$compatible set $A$. Let $A_{i}=\left\{\left.\alpha\right|_{V_{i}}: \alpha \in A\right\}$, where $V_{i}$ are vertex sets of $G_{i}$ for $i=1,2$. It is clear that $A_{i}$ is an $H_{G_{i}}$-compatible set for each $i=1,2$. We construct a bipartite graph $M$ whose vertex set corresponds to $A_{1}$ and $A_{2}$, where an edge is given between two members $\beta \in A_{1}$ and $\gamma \in A_{2}$ if and only if $\beta \oplus \gamma \in A$. We claim that the $M$ is a forest. From this claim, it is straightforward to see that $\nu(G) \leq \nu\left(G_{1}\right)+\nu\left(G_{2}\right)-1$, since $|A|$ is the number of edges in $M$.

In order to prove the claim, consider the endpoint $v_{1}$ of the bridge $e$ in $G_{1}$. We construct a shortest-path tree $T$ from $v_{1}$ in $G_{1}$, and give the breadth-first ordering $v_{1}, v_{2}, \ldots, v_{t}$ of vertices of $G_{1}$ along this tree. This ordering is a pathpreserving ordering of $T$, although it may not be a path-preserving ordering of $G_{1}$. Let $U^{j}=\left\{v_{1}, v_{2}, \ldots, v_{j}\right\}$, and let $A_{1}^{j}$ be $\left.A_{1}\right|_{U^{j}}$. We consider a bipartite graph $M_{j}$ whose vertex set corresponds to $A_{1}^{j}$ and $A_{2}$, where an edge is given between two members $\beta^{j} \in A_{1}^{j}$ and $\gamma \in A_{2}$ if and only if there exists $\beta \in A_{1}$ such that $\beta^{j}=\left.\beta\right|_{U^{j}}$ and $\beta \oplus \gamma \in A$.

It suffices to show that $M_{j}$ is a forest for every $j$, since $M_{t}=M$. The graph $M_{0}$ is defined to be a star graph connecting all the nodes corresponding to assignments in $A_{2}$ to a node (representing the empty assignment). We can construct $M_{j}$ from $M_{j-1}$ by splitting each node $x(\alpha)$ corresponding to an 
assignment in $\alpha \in A_{1}^{j-1}$ into two nodes $x(\alpha \oplus 0)$ and $x(\alpha \oplus 1)$, one assigns 0 and the other assigns 1 to $v_{j}$. The neighbors of $x(\alpha)$ is connected to $x(\alpha \oplus 0)$ and/or $x(\alpha \oplus 1)$ by definition.

Analogously to the proof of Lemma 3.1, we show that at most one neighbor of $x$ can be connected to both of $x(\alpha \oplus 0)$ and $x(\alpha \oplus 1)$. Assume on the contrary that there exist $\gamma \neq \gamma^{\prime} \in A_{2}$ such that both of the corresponding nodes $y(\gamma)$ and $y\left(\gamma^{\prime}\right)$ are adjacent to both of $x(\alpha \oplus 0)$ and $x(\alpha \oplus 1)$. From the adjacency relation, there exists $\beta_{0}, \beta_{1}, \beta_{2}, \beta_{3}$ such that all of $\beta_{0} \oplus \gamma, \beta_{1} \oplus \gamma, \beta_{2} \oplus \gamma^{\prime}$, and $\beta_{3} \oplus \gamma^{\prime}$ are in $A$, and the restriction of $\beta_{i}$ to $U^{j}$ is $\alpha \oplus 0$ if $i$ is even, otherwise $\alpha \oplus 1$.

Let $u$ be the nearest vertex in $V_{2}$ from the bridge $e$ satisfying that $\gamma(u) \neq \gamma^{\prime}(u)$. We can assume that $\gamma(u)=0$ and $\gamma^{\prime}(u)=1$. Since $T$ is the shortest path tree, at least one shortest path (in $G$ ) between $u$ and $v_{j}$ is a path in $T \cup G_{2}$. On the path, the entry sums of $\beta_{0} \oplus \gamma$ and $\beta_{3} \oplus \gamma^{\prime}$ differs by two from each other. This contradicts the property of a compatible set.

Thus, we have shown that at most one neighbor of $x$ can be connected to both of $x(\alpha \oplus 0)$ and $x(\alpha \oplus 1)$. If $M_{j-1}$ is a forest. we can see that such a splitting operation keeps the graph to be a forest, and accordingly, $M_{j}$ is a forest. Thus, we can prove the claim by induction.

The affine independence also follows from this claim in a routine way: Suppose that $A$ does not satisfy the affine independence. Then, there exists real numbers $c_{\alpha}$ for $\alpha \in A$ such that $\sum_{\alpha \in A} c_{\alpha}=0, \sum_{\alpha \in A} c_{\alpha} \alpha=\mathbf{0}$, and at least one $c_{\alpha}$ is nonzero. Because of affine independence of $A_{1}, \sum_{\alpha,\left.\alpha\right|_{V_{1}}=\beta} c_{\alpha}=0$ for each fixed $\beta \in A_{1}$. Similarly, $\sum_{\alpha,\left.\alpha\right|_{V_{2}}=\gamma} c_{\alpha}=0$ for each fixed $\gamma \in A_{2}$.

Let us take a leaf node $z$ in $M$. Without loss of generality, we assume $z$ corresponds to a member $\beta$ of $A_{1}$. Then, we can conclude that $c_{\alpha}=0$ for the unique member $\alpha$ of $A$ such that $\left.\alpha\right|_{V_{1}}=\beta$. We remove $z$ from $M$ and continue this process iteratively to see that $c_{\alpha}=0$ for all $\alpha \in A$.

A graph $G$ is series connection of two graphs $G_{1}$ and $G_{2}$ if $G=G_{1} \cup G_{2}$ and $G_{1} \cap G_{2}=\{v\}$ (implying that they share no edge), where $v$ is called the separator. We have the following:

Theorem 4.4 Suppose that a graph $G$ is a series connection of two connected graphs $G_{1}$ and $G_{2}$, and let $v$ be the separator. Then, we have the following: (1) If there is an $H_{G}$ compatible set $A$ such that $|A|=\nu(G)$ and there exist $\alpha$ and $\beta$ in $A$ satisfying $\alpha(v) \neq \beta(v), \nu(G) \leq \nu\left(G_{1}\right)+\nu\left(G_{2}\right)-2$.

(2) Otherwise, let $\nu^{0}\left(G_{i}\right)$ be the maximum size of a compatible set $A_{i}$ for $H_{G_{i}}$ such that $\alpha(v)=0$ for every $\alpha \in A_{i}$. Then, $\nu(G) \leq \nu^{0}\left(G_{1}\right)+\nu^{0}\left(G_{2}\right)-1$.

(3)If both of $H_{G_{1}}$ and $H_{G_{2}}$ satisfy the affine independence property, so does 
$H_{G}$.

Proof: Consider an $H_{G^{-}}$compatible set $A$. Let $A_{i}=\left\{\left.\alpha\right|_{V_{i}}: \alpha \in A\right\}$, where $V_{i}$ are vertex sets of $G_{i}$ for $i=1,2$. It is clear that $A_{i}$ is an $H_{G_{i}}$-compatible set for each $i=1,2$. Let $A^{0}=\{\alpha \in A: \alpha(v)=0\}$ and $A^{1}=\{\alpha \in A: \alpha(v)=1\}$.

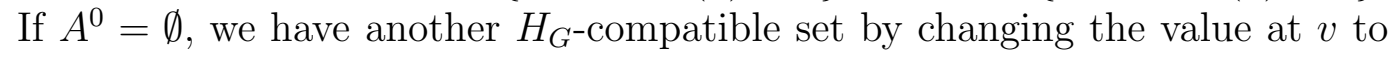
be 0 for all elements in $A$. Thus, we can assume that $A^{0} \neq \emptyset$.

Let $A_{i}^{0}=\left\{\alpha \in A_{i}: \alpha(v)=0\right\}$ and $A_{i}^{1}\left\{\alpha \in A_{i}: \alpha(v)=1\right\}$ for $i=1,2$. Then, analogous to the proof of Theorem 4.3, we can construct a forest to prove that $\left|A^{0}\right| \leq\left|A_{1}^{0}\right|+\left|A_{2}^{0}\right|-1$. Similarly, $\left|A^{1}\right| \leq\left|A_{1}^{1}\right|+\left|A_{2}^{1}\right|-1$ if $A^{1} \neq \emptyset$. Thus, we have (1) and (2).

(3) can be proved analogously to Theorem 4.3.

\subsection{The Case of a Cycle}

Let $C_{n}$ be a cycle on $n$ vertices $V=\{1,2, \ldots, n\}$ with edge set $\left\{e_{1}, \ldots, e_{n}\right\}$ where $e_{i}=(i, i+1), 1 \leq i \leq n$. The arithmetics on vertices are cyclic, i.e., $n+1=1$. We sometimes refer to the edge $e_{n}$ as $e_{0}$ as well. For $i, j \in V$, let $P(i, j)$ denote the path from $i$ to $j$ containing the nodes $v_{i}, v_{i+1}, \ldots v_{j}$ in this cyclic order. Note that $P(j, i)$ is different from $P(i, j)$ if $i \neq j . P(i, i)$ is naturally interpreted as an empty path consisting of a single vertex and no edge. Let $\mathcal{P}=\mathcal{P}_{C_{n}}$ be a set of shortest paths on $C_{n}$. Note that for any given edge lengths, $\mathcal{P}$ satisfies the following conditions: (1) $P(i, i) \in \mathcal{P}$ for every $i \in V$, (2) if $P \in \mathcal{P}$ then every subpath of $P$ is in $\mathcal{P}$, and (3) for every pair $i, j$ of distinct vertices of $C_{n}$, at least one of $P(i, j)$ and $P(j, i)$ is in $\mathcal{P}$.

We can also assume that $P(i, i+1)$ is always a shortest path between $v_{i}$ and $v_{i+1}$; otherwise, $e_{i}$ is contained in no shortest path, and we can simply remove the edge $e_{i}$ from the consideration to reduce the problem to the case where the graph is a path.

Theorem $4.5 \nu\left(H_{C_{n}}\right)=n+1$.

A graph $G$ is a tree of cycles if either (1) $G$ is a tree or (2) $G$ has a proper subgraph $G^{\prime}$ that is a tree of cycles and a subgraph $C$ that is either a cycle or an edge such that $G$ is obtained by series connection of $G^{\prime}$ and $C$.

As a corollary of Theorem 4.5 and Theorem 4.4, we have the following:

Corollary $4.6 \nu\left(H_{G}\right)=n+1$ if $G$ is a tree of cycles with $n$ vertices.

Proof: We prove by induction on the number of vertices. If $G$ is a tree, we 
have already proven that the statement holds. Suppose that $G$ is obtained as a series connection of $G^{\prime}$ and $C$. We focus on the case where $C$ is a cycle, since it is easy to handle the case where $C$ itself is an edge. Let $n_{1}$ and $n_{2}$ be number of vertices in $G^{\prime}$ and $C$, respectively. By definition, $n=n_{1}+n_{2}-1$ is the number of vertices in $G$ and $n_{1}<n$.

If $\nu(G) \leq \nu\left(G^{\prime}\right)+\nu(C)-2$, we have $\nu(G) \leq n_{1}+1+n_{2}+1-2=n+1$.

Otherwise, $\nu(G) \leq \nu^{0}\left(G^{\prime}\right)+\nu^{0}(C)-1$. We consider a cycle $C^{\prime}$ with $n_{2}-$ 1 vertices by replacing the joint node $v$ in $C$ and its adjacent edges $e=$ $(\operatorname{prev}(v), v)$ and $e^{\prime}(v, \operatorname{succ}(v))$ by an edge $e^{\prime \prime}=(\operatorname{prev}(v), \operatorname{succ}(v))$ whose edge weight is the sum of those of $e$ and $e^{\prime}$. Then, it is routine to verify that $\nu^{0}(C)=\nu\left(C^{\prime}\right)$. Thus, we have $\nu(G) \leq n_{1}+1+n_{2}-1=n+1$.

We often write $\mathcal{P}$ for $H_{C_{n}}$ identifying the hypergraph and the set of hyperedges in this section for abbreviation. We devote the rest of this section for proving Theorem 4.5.

For $n \leq 2$ the theorem is trivial to verify, so we will assume $n \geq 3$ in the sequel. For an assignment $\alpha$, we define $w(\alpha)=w_{V}(\alpha)=\sum_{v \in C_{n}} \alpha(v)$ to be the weight of $\alpha$ over all vertices in $C_{n}$.

Lemma 4.7 Let $\alpha$ and $\beta$ be $\mathcal{P}$-compatible assignments on $C_{n}$. Then, $w(\alpha)$ and $w(\beta)$ differ by at most 1.

Proof: Suppose that assignments $\alpha$ and $\beta$ are $\mathcal{P}$-compatible and $w(\beta) \geq$ $w(\alpha)+2$. Let's say that each vertex $v$ has type $(\alpha(v), \beta(v))$. Cyclically list the vertices of types $(0,1)$ and $(1,0)$ in the direction of the cycle, ignoring those of types $(0,0)$ or $(1,1)$. Then, since the number of vertices of type $(0,1)$ is greater than that of the vertices of type $(1,0)$ by at least 2 , there are at least two places where type $(0,1)$ vertices appear consecutively. At least one of such consecutive pairs forms (together with $(0,0)$ and $(1,1)$ entries between the vertices of the pair) a path in $\mathcal{P}$, on which $\alpha$ and $\beta$ are not compatible.

Lemma 4.8 Suppose $w(\alpha)=w(\beta)$ for assignments $\alpha$ and $\beta$. Then, if $\alpha$ and $\beta$ are $\mathcal{P}$-compatible they are compatible on every path of $C_{n}$.

Proof: We show that $\alpha$ and $\beta$ are compatible on an arbitrary path $P(i, j)$. If $i=j+1$ then the path consists of all the vertices of $C_{n}$ and, because $\alpha$ and $\beta$ are of the same weight, they are compatible on $P(i, j)$. Suppose $i \neq j+1$. Then, path $P(j+1, i-1)$ is the complement of path $P(i, j)$ in terms of their vertex sets. At least one of $P(i, j)$ and $P(j+1, i-1)$ is in $\mathcal{P}$ and hence $a$ and $b$ are compatible on at least one of these paths. But the compatibility on one of these paths implies the compatibility on the other, since the vertex 
sets of these paths are the complement of each other. Therefore $\alpha$ and $\beta$ are compatible on $P(i, j)$.

From the above observations and Corollary 4.2, it is clear that $\nu\left(H_{C_{n}}\right) \leq$ $2(n+1)$. We need some more tools in order to sharpen this bound.

The following notion of edge opposition is one of our main tools. Let $e_{i}$ and $e_{j}$ be two edges of $C_{n}$. We say $e_{i}$ opposes $e_{j}$ (and vice versa) if paths $P(i+1, j)$ and $P(j+1, i)$ are both in $\mathcal{P}$. Note that when $P(i+1, i) \in \mathcal{P}, e_{i}$ opposes itself in this definition. However, in this case, the length of $e_{i}$ is so large that it does not appear in any shortest path, and we can cut the cycle into a path at $e_{i}$ to reduce the problem into the sequence rounding problem. Thus, we assume this does not happen.

Lemma 4.9 For every edge $e_{i}$ of $C_{n}$, there is at least one edge $e_{j}$ that opposes $e_{i}$.

Proof: Fix an edge $e_{i}$. Let $P$ be the maximal path in $\mathcal{P}$ ending at $i$ and let $P^{\prime}$ be the maximal path in $\mathcal{P}$ starting at $i+1$. We claim that $V(P) \cup V\left(P^{\prime}\right)=$ $V\left(C_{n}\right)$ : if there is some $k$ in neither $P$ nor $P^{\prime}$, then neither $P(k, i)$ nor $P(i, k)$ is in $\mathcal{P}$, contradicting the definition of the shortest path system. Therefore, there is an edge $e_{j}$ such that $j \in V\left(P^{\prime}\right)$ and $j+1 \in V(P)$. These conditions imply $P(i+1, j) \in \mathcal{P}$ and $P(j+1, i) \in \mathcal{P}$, that is, $e_{j}$ opposes $e_{i}$.

Lemma 4.10 If edges $e_{i}$ and $e_{j}$ oppose each other, then, either $e_{i+1}$ opposes $e_{j}$ or $e_{j+1}$ opposes $e_{i}$.

Proof: We start with the special case where $i+1=j$. Since $n \geq 3, j+1 \neq i$ in this case. Since $P(j+1, i) \in \mathcal{P}, \mathcal{P}$ also contains $P(j+2, i)$, which is welldefined because $j+1 \neq i$. From our assumption on the graph $C_{n}, P(j, j+1)=$ $P(i+1, j+1) \in \mathcal{P}$; thus $e_{j+1}$ opposes $e_{i}$. The case where $j+1=i$ is similar, so assume $i+1 \neq j$ and $j+1 \neq i$. Then, we have both $P(j+2, i)$ and $P(i+2, j)$ in $\mathcal{P}$ similarly to the above. Therefore, if $P(i+1, j+1) \in \mathcal{P}$ then $e_{i+1}$ opposes $e_{j}$. Otherwise, $P(j+1, i+1) \in \mathcal{P}$ and therefore $e_{j+1}$ opposes $e_{i}$.

Define the opposition graph, denoted by $\operatorname{opp}(\mathcal{P})$, to be the graph on $E\left(C_{n}\right)$ in which $\left\{e_{i}, e_{j}\right\}$ is an edge if and only if $e_{i}$ and $e_{j}$ oppose each other. By Lemma 4.9 and Lemma 4.10, we obtain the following:

Lemma 4.11 The opposition graph opp $(\mathcal{P})$ is connected.

We next prove a lemma regarding two equivalence relations on the vertex set of a graph. Let $G$ be a graph. We say that a pair $\left(R_{1}, R_{2}\right)$ of equivalence relations on $V(G)$ honors $G$, if for every edge $\{u, v\}$ of $G, u$ and $v$ are equivalent either in $R_{1}$ or in $R_{2}$. For an equivalence relation $R$, denote by $e c(R)$ the number of 
equivalence classes of $R$.

Lemma 4.12 Let $G$ be a connected graph on $n$ vertices and suppose a pair $\left(R_{1}, R_{2}\right)$ of equivalence relations on $V(G)$ honors $G$. Then ec $\left(R_{1}\right)+\operatorname{ec}\left(R_{2}\right) \leq$ $n+1$.

Proof: Fix an arbitrary spanning tree $T$ of $G$. We assume $\left(R_{1}, R_{2}\right)$ honors $G$ and hence it honors $T$. We grow tree $S$ from a singleton tree towards $T$, and consider $f(S)$ that is the sum of the number of equivalence classes for $R_{1}$ and $R_{2}$ among the nodes of $S$. Initially, we have one node, and hence $f(S)=2$. If we add an edge and a vertex, $f(S)$ increases by at most one, since the vertex is equivalent to the vertex it attached to for at least one of the equivalence relations. Hence, $f(T) \leq n+1$.

A $\mathcal{P}$-compatible set $A$ is called uniform if there is a fixed integer $w$ such that $w(\alpha)=w$ for every $\alpha \in A$. We call $w$ the weight of $A$.

The following equivalence relation on the edge set of $C_{n}$ plays a central role in our proof.

Let $A$ be a uniform $\mathcal{P}$-compatible set. We say that two edges $e_{i}, e_{j}$ of $C_{n}$ are $A$-equivalent and write $e_{i} \sim_{A} e_{j}$ if and only if either $i=j$ or $w_{P(i+1, j)}(\alpha)$ is the same for every $\alpha \in A$. This relation is symmetric since the assignments in $A$ have the same weight on the entire cycle and $P(i+1, j)$ and $P(j+$ $1, i)$ are complement to each other in terms of their vertex sets. It is indeed straightforward to check the transitivity to confirm that $A$-equivalence is an equivalence relation.

Lemma 4.13 Let $A$ and $B$ be uniform $\mathcal{P}$-compatible sets on $C_{n}$ such that $A \cup B$ is a $\mathcal{P}$-compatible set. We assume that $A$ and $B$ has weights $w$ and $w+1$, respectively. Then, for any pair of edges $e_{i}$ and $e_{j}$ opposing each other with respect to $\mathcal{P}$, either $e_{i} \sim_{A} e_{j}$ or $e_{i} \sim_{B} e_{j}$; in other words, the pair $\left(\sim_{A}, \sim_{B}\right)$ honors the opposition graph opp $(\mathcal{P})$.

Proof: Let $A, B, e_{i}, e_{j}$ be as in the statement of the lemma and suppose that neither $e_{i} \sim_{A} e_{j}$ nor $e_{i} \sim_{B} e_{j}$ holds. Since $e_{i}$ is not $A$-equivalent to $e_{j}$, there are $\alpha_{1}, \alpha_{2} \in A$ such that $w_{P(i+1, j)}\left(\alpha_{1}\right)<w_{P(i+1, j)}\left(\alpha_{2}\right)$. Similarly, there are $\beta_{1}, \beta_{2} \in$ $B$ such that $w_{P(i+1, j)}\left(\beta_{1}\right)<w_{P(i+1, j)}\left(\beta_{2}\right)$. First suppose that $w_{P(i+1, j)}\left(\alpha_{2}\right) \leq$ $w_{P(i+1, j)}\left(\beta_{1}\right)$. Then we have $w_{P(i+1, j)}\left(\beta_{2}\right) \geq w_{P(i+1, j)}\left(\alpha_{1}\right)+2$, and hence $\alpha_{1}$ and $\beta_{2}$ are incompatible on path $P(i+1, j)$. This is a contradiction because, since $e_{i}$ and $e_{j}$ are opposing each other, path $P(i+1, j)$ must be in $\mathcal{P}$. Next suppose $w_{P(i+1, j)}\left(\alpha_{2}\right)>w_{P(i+1, j)}\left(\beta_{1}\right)$. Since $w\left(\beta_{1}\right)=w\left(\alpha_{2}\right)+1$, we then have $w_{P(j+1, i)}\left(\beta_{1}\right) \geq w_{P(j+1, i)}\left(\alpha_{2}\right)+2$, the incompatibility of $\beta_{1}$ and $\alpha_{2}$ on path $P(j+1, i) \in \mathcal{P}$. 
Consider a uniform $\mathcal{P}$-compatible set $A$. From Lemma 4.8, $A$ is an $I_{n}$-compatible set, where $I_{n}$ is the hypergraph on $V$ associated with all the intervals on the graph obtained by cutting $C_{n}$ at the edge $e_{0}=\left(v_{n}, v_{1}\right)$. Let $V_{i}=\{1,2, \ldots, i\} \subset$ $V$, and let $A\left(V_{i}\right)$ be the set of assignments on $V_{i}$ obtained by restricting $A$ to $V_{i}$.

Lemma 4.14 $\left|A\left(V_{i}\right)\right| \leq\left|A\left(V_{i-1}\right)\right|+1$.

Proof: $V_{i}=V_{i-1} \cup\left\{v_{i}\right\}$. Applying Lemma 3.1, there is at most one assignment $\alpha$ in $A\left(V_{i-1}\right)$ such that both of $\alpha \oplus 0$ and $\alpha \oplus 1$ are in $A\left(V_{i}\right)$. Thus, we obtain the lemma.

We call the index $i$ a branching index of $A$ if $\left|A\left(V_{i}\right)\right|=\left|A\left(V_{i-1}\right)\right|+1$ holds. Note that for a branching index, there must be an assignment $\alpha$ in $A\left(V_{i-1}\right)$ such that both of $\alpha \oplus 0$ and $\alpha \oplus 1$ are in $A\left(V_{i}\right)$.

Lemma 4.15 Let $A$ be a uniform $\mathcal{P}$-compatible set. Then, $i$ is a branching index in $A$ only if the edge $e_{i}=(i, i+1)$ is A-equivalent to none of $e_{0}, e_{1}, e_{2}, \ldots, e_{i-1}$.

Proof: Suppose level $i$ is a branching index. Then, we have $\alpha \in A\left(V_{i-1}\right)$ such that $\alpha \oplus 0$ and $\alpha \oplus 1$ are in $A\left(V_{i}\right)$. If $e_{i}$ is $A$-equivalent to $e_{j}$ for $j<i$, the assignments $\alpha \oplus 0$ and $\alpha \oplus 1$ must have the same total weight on $V_{i} \backslash V_{j}=$ $\{j+1, \ldots, i\}$. This is impossible, since two assignments are the same on $V_{i} \backslash V_{j}$ except on $i$.

Consider the number ec $\left.\left(\sim_{A}\right)\right|_{V_{i}}$ of equivalence classes in $V_{i}$. Lemma $4.15 \mathrm{im}$ plies that $\left|A\left(V_{i}\right)\right|-\left|A\left(V_{i-1}\right)\right| \leq\left. e c\left(\sim_{A}\right)\right|_{V_{i}}-\left.e c\left(\sim_{A}\right)\right|_{V_{i-1}}$. Thus, we have the following corollary:

Corollary 4.16 Let $A$ be a uniform $\mathcal{P}$-compatible set. Then $|A| \leq e c\left(\sim_{A}\right)$.

We are now ready to prove Theorem 4.5. Let $\mathcal{P}$ be a shortest path system on $C_{n}$ and let $A$ be a $\mathcal{P}$-compatible set on $C_{n}$. By Lemma 4.7, the assignments of $A$ have at most two weights. If there is only one weight, then $|A| \leq e c\left(\sim_{A}\right)$ by Corollary 4.16 and hence $|A| \leq n+1$. Suppose $A$ consists of two subsets $A_{1}$ and $A_{2}$, with the assignments in $A_{1}$ having weight $w$ and those in $A_{2}$ having weight $w+1$. By Lemma 4.13 , the pair of equivalence relations $\left(\sim_{A_{1}}, \sim_{A_{2}}\right)$ honors the opposition graph $\operatorname{opp}(\mathcal{P})$. Since $\operatorname{opp}(\mathcal{P})$ is connected (Lemma 4.11), we have $e c\left(\sim_{A_{1}}\right)+e c\left(\sim_{A_{2}}\right) \leq n+1$ by Lemma 4.12 . We are done, since $\left|A_{i}\right| \leq e c\left(\sim_{A_{i}}\right)$ for $i=1,2$ by Corollary 4.16 . 


\section{Concluding remarks}

We have proven the conjectures only for special graphs. It will be nice if the conjectures are proven for wider classes of graphs such as series parallel graphs ${ }^{3}$. Also, the affine independence property for the cycle graph has not been proven in this paper.

For a general graph, we do not even know whether $\nu\left(H_{G}\right)$ is polynomially bounded by the number of vertices. It is plausible that the number of roundings can become large if the entries have some middle values (around 0.5). For a special input a consisting of entries with a same value $0.5+\epsilon$, we can show that the number of global roundings of $\mathbf{a}$ is bounded by $n+1$ if $G$ is bipartite; otherwise by $m+1$, where $m$ is the number of edges in $G$ [9].

Acknowledgement. The authors thank Jesper Jansson, Günter Rote, and Akiyoshi Shioura for fruitful discussion.

\section{References}

[1] T. Asano, N. Katoh, K. Obokata, and T. Tokuyama, Matrix Rounding under the $L_{p}$-Discrepancy Measure and Its Application to Digital Halftoning, Proc. 13th ACM-SIAM Symp. on Discrete Algorithms (SODA2002), 2002, pp. 896904.

[2] T. Asano, T. Matsui, and T. Tokuyama, Optimal Roundings of Sequences and Matrices, Nordic Journal of Computing 7, 2000, pp.241-256.

[3] T. Asano and T. Tokuyama, How to Color a Checkerboard with a Given Distribution - Matrix Rounding Achieving Low $2 \times 2$ Discrepancy, Proc. 12th Int'l. Symp. on Algorithms and Computation (ISAAC2001) LNCS 2223, 2001, pp. 636-648.

[4] J. Beck and V. T. Sós, Discrepancy Theory, ine T. Graham, M. Grötshel, and L. Lovász (Eds.) Handbook of Combinatorics, Vol. II, Elsevier Sciences, 1995, Chapter 26, pp. 1405-1446.

[5] B. Bollobás. Modern Graph Theory, GTM 184, Springer-Verlag, 1998.

[6] B. Chazelle, The Discrepancy Method: Randomness and Complexity, Princeton University Press, 2000.

[7] B. Doerr, Lattice Approximation and Linear Discrepancy of Totally Unimodular Matrices, Proc. 12th ACM-SIAM Symp. on Discrete Algorithms (SODA2001), 2001, pp.119-125.

$\overline{3}$ One of the authors recently proved it for outerplanar graphs [14] 
[8] A. Hoffman and G. Kruskal, Integral Boundary Points of Convex Polyhedra, In W. Kuhn and A. Tucker (Eds.) Linear Inequalities and Related Systems, 1956, pp. 223-246.

[9] J. Jansson and T. Tokuyama, Semi-Balanced Coloring of Graphs-2-Colorings Based on a Relaxed Discrepancy Condition, to appear in Graphs and Combinatorics. Preliminary version is available as a Master thesis of J. Jansson at http://www.comp.nus.edu.sg/ jansson/Thesis_MSc_Math.html.

[10] J. Matoušek, Geometric Discrepancy, Algorithms and Combinatorics 18, Springer Verlag 1999.

[11] H. Niederreiter, Random Number Generations and Quasi Monte Carlo Methods, CBMS-NSF Regional Conference Series in Applied Math., SIAM, 1992.

[12] J. Pach and P. Agarwal, Combinatorial Geometry, John-Wiley \& Sons, 1995.

[13] K. Sadakane, N. Takki-Chebihi, and T. Tokuyama, Combinatorics and Algorithms on Low-Discrepancy Roundings of a Real Sequence, Proc. 28th Int'l, Colloquium on Automata, Languages, and Programming (ICALP2001), LNCS 2076, 2001, pp. 166-177.

[14] N. Takki-Chebihi and T. Tokuyama, Enumerating Global Roundings of an outerplanar graph, Proc. Int'l. Symp. on Algorithms and Computation (ISAAC2003), LNCS 2906, 2003, pp. 425-433. 